\title{
Author Correction: Focal molography is a new method for the in situ analysis of molecular interactions in biological samples
}

Volker Gatterdam, Andreas Frutiger, Klaus-Peter Stengele, Dieter Heindl, Thomas Lübbers, Janos Vörös and Christof Fattinger

Correction to: Nature Nanotechnology https://doi.org/10.1038/nnano.2017.168, published online 25 September 2017.

In the Supplementary Information file for this Article, in Supplementary Fig. 7a, it was unnecessary to state the outcoupling periods, indicated by the striated pattern. In Supplementary Fig. 8c, the range of the outcoupling periods, shown by the striated pattern, was incorrect as was the associated label, and the labels $-90^{\circ}, 0^{\circ}$ and $90^{\circ}$ were missing. The caption for this figure has been updated accordingly. The amended Supplementary Information file is supplied with this correction notice. The original file has not been corrected.

\section{Additional information}

Supplementary information The online version contains supplementary material available at https://doi.org/10.1038/s41565-021-00863-x.

Published online: 11 February 2021

https://doi.org/10.1038/s41565-021-00863-X

(c) The Author(s), under exclusive licence to Springer Nature Limited 2021 